\title{
Reflexões Sobre a Tecnologia e Adolescentes: Mitos e Verdades
}

\author{
Daniela Krone Dominski ${ }^{(1)}$, Débora Emerim Nunes Brito ${ }^{(2)}$, Idegar Nunes dos Santos ${ }^{(3)}$, Juliana Alves Rodrigues ${ }^{(4)}$, \\ Eliane Moura $^{(5)}$, Regina Maria Fernandes Lopes ${ }^{(6)}$, Cristiane Silva Esteves ${ }^{(7)}$
}

\begin{abstract}
Resumo: O avanço tecnológico na área da informação e comunicação vem interferindo diretamente nas atividades comunicativas humanas. Este artigo aborda os mitos e verdades da relação entre a tecnologia e os adolescentes estudantes e objetiva analisar o uso excessivo da Internet por adolescentes. O método utilizado para este estudo foi indutivo e as características apresentadas apontam que o tipo de pesquisa utilizada de revisão de literatura, referente à relação do hábito de leitura e a internet, o uso adequado para acessá-la, influencia no comportamento dos adolescentes, as vantagens e desvantagens da utilização da tecnologia na educação dos estudantes adolescentes. Após a análise dos dados, buscou-se levantar os determinantes da ocorrência desse comportamento, verificar os sites mais utilizados, constatar o tempo de uso e levantar os possíveis riscos desse uso abusivo, bem como dar subsídio a novas pesquisas para se construir uma melhor forma de abordar o problema. Os resultados mostraram, qualitativamente a relevância positiva que a internet, dentre outras tecnologias, tem apresentado, abrindo um mundo de possibilidades aos estudantes da era digital. Pesquisas recentes mostraram que o cyberbullying, tem sido denominada na literatura científica, esta nova forma de assédio realizada através da tecnologia, vem trazendo prejuízos para a população, principalmente para os pares de adolescentes.
\end{abstract}

Palavras-Chave: Adolescência; Estudantes; Internet; dependência internet; cyberbullying.

\section{Reflections On Technology And Teens: Myths And Truths}

\begin{abstract}
Technological advances in information and communication is directly interfering in human communicative activities. This article discusses the myths and truths of the relationship between technology and young students and analyzes the excessive use of Internet by adolescents. The method used for this study was inductive and the characteristics presented show that the type of research used literature review, concerning the relationship of reading habits and the internet, the proper use to access it, influences the behavior of adolescents, the advantages and disadvantages of using technology in the education of adolescent students. After analyzing the data, we sought to raise the determinants of the occurrence of this behavior, check the sites most commonly used, note the time of use and raise the risk of possible abuse, as well as give support to new research to build a better way to address the problem. The results showed qualitatively the positive significance that the Internet, among other technologies, is presented, opening a world of possibilities for students in the digital age. Recent research has shown that cyberbullying has been named in the scientific literature, this new form of harassment carried out by technology, has brought to the local people, especially for pairs of adolescents.
\end{abstract}

Key-words: Adolescence; Students, Internet, Internet addiction, cyberbullying,

\footnotetext{
${ }^{1}$ Daniela Dominski é Acadêmica de Administração de empresas do CESUCA- Faculdade INEDI. Cachoeirinha - RS. Brasil; ${ }^{2}$ Débora Brito é Acadêmica de Administração de empresas do CESUCA- Faculdade INEDI. Cachoeirinha - RS. Brasil; ${ }_{3}^{3}$ Idegar Nunes é Acadêmico de Administração de empresas do CESUCA- Faculdade INEDI. Cachoeirinha - RS. Brasil;

${ }^{4}$ Juliana Alves é Acadêmica de Administração de empresas do CESUCA- Faculdade INEDI. Cachoeirinha - RS. Brasil;

${ }^{5}$ Eliane Moura é Professora do CESUCA- Faculdade INEDI. Disciplina de Metodologia da Pesquisa e Seminário. Brasil;

${ }^{6}$ Cristiane Silva Esteves é Graduada em Psicologia (PUC/RS), Mestranda em psicologia Clínica pela mesma IES. Pós-graduanda em Atendimento clínico, ênfase em psicanálise (UFRGS). E-mail: crissilvaesteves@ gmail.com;

${ }^{7}$ Regina Maria Fernandes Lopes é Psicóloga (PUC/RS). Mestre em Psicologia (PUC/RS). Especialista em psicologia Clínica Ênfase em Avaliação Psicológica (UFRS). Coordenadora do Núcleo Médico-Psicológico. Porto Alegre - RS. Brasil. Email: reginamlopess@gmail.com.
} 


\section{Introdução}

O avanço tecnológico na área da informação e comunicação vem interferindo diretamente nas atividades comunicativas humanas. Se, antes da década de 90, de modo geral, a forma mais rápida de comunicação envolvendo tecnologia versava sobre o uso do telefone fixo, a partir de 1990, com o advento da Internet nos deparamos com um recurso inovador (COSTA, 2005; MARCUSCHI, apud GOMES, A. L. et al 2005). Através desta ferramenta, passamos a interagir com mais rapidez, podendo inclusive conversar virtualmente com uma ou mais pessoas ao mesmo tempo, além de obter acesso a uma fonte com uma gama imensa de informações consultando à rede World Wide Web (www).

O contínuo progresso tecnológico provê cada vez mais facilidade de acesso à Internet, através de diferentes suportes, como aparelhos celulares, notebooks, tablets, ipad, iphone e outros. Estes suportes eletrônicos portáteis permitem ao usuário se conectar à Internet a qualquer hora e qualquer lugar, tornando cada vez mais intensa a relação que se estabelece entre tecnologia e comunicação, permitindo a ele entrar em contato com novas formas de ação através da linguagem, como as listas de discussão e chat aberto, associadas ao contexto da cultura eletrônica (EISENKRAEMER, 2006).

Alguns gêneros textuais vinculados ao contexto digital tomam como referência os gêneros textuais já existentes (COSTA, 2005; MARCUSCHI, apud GOMES, Ana A.L. et al 2005). Por isso, encontramos semelhanças entre as formas de alguns destes gêneros digitais e os tradicionais, como se observa ocorrer entre o e-mail e a carta, as mensagens deixadas no Orkut e os bilhetes, ou, ainda, o blog e o diário pessoal (PEREIRA \& MOURA, 2005).

Para conversarem através da Internet, os adolescentes usam vários tipos de gêneros de texto digitais. Com frequiência, seus textos vêm sendo produzidos de modo alternativo e casual, utilizando a linguagem escrita de forma mais simplificada e com várias transgressões, como iniciar frases com letra minúscula ou, então, não acentuar as palavras e atém mesmo abreviá-las. A forma não-convencional de escrever na Internet transformou-se em um modismo entre os internautas, incluindo os mais jovens, caracterizando, assim, a escrita adolescente nos canais digitais de comunicação (COSTA, 2005; BOLTER, 2001; LEE, 2007).

Essa inovação tecnológica gera um grande questionamento dos pais e educadores referente à relação que os mesmos estão desenvolvendo com o mundo virtual. Com isso, surgem dúvidas sobre a influência que a internet poderá exercer sobre o comportamento dos adolescentes; se é necessário estipular um tempo limite de acesso a rede, dentre mitos e curiosidades desta relação Internet X Adolescentes.

Id en line Revista de Psicologia. Ano 7, No. 20, Julho/2013 - ISSN 1981-1179.

Edição eletrônica em http://idonline.emnuvens.com.br/id 


\section{Método}

As características apresentadas neste artigo apontam que o tipo de pesquisa utilizada é a bibliográfica, tendo em vista que toda a pesquisa é bibliográfica em seu primeiro momento, pois busca fornecer considerações teóricas para elucidar alguns conceitos básicos. Desta forma, apresenta-se uma revisão da bibliografia mais recente sobre os mitos e verdades da relação entre a tecnologia e os adolescentes. Segundo Gil (2007, p.65), "a pesquisa bibliográfica é desenvolvida a partir de material já elaborado, constituído principalmente de livros e artigos científicos". Tendo como objetivo analisar o uso excessivo da Internet por adolescentes, o estudo baseou-se em uma revisão da literatura a partir do levantamento de artigos no Medline/PubMed, PsycINFO, Google Acadêmico e livros publicados nos últimos anos.

\section{Resultados}

\section{O hábito de leitura e a internet}

Mesmo com o avanço tecnológico, ainda há divergências populares de opiniões a respeito da possibilidade de que os adolescentes percam ou até mesmo não desenvolvam o hábito da leitura devido à utilização da internet. Dentre as referências consultadas, ficou constatado que isso é um engano, tendo em vista que os mesmos acabam lendo mais quando buscam assuntos de seus próprios interesses.

Quando uma escola conecta-se a Internet, um novo mundo de possibilidades se abre diante de alunos e professores. Não mais falamos, a partir daí, dos instrumentos didáticos como um livro ou uma enciclopédia; falamos de uma infinidade de livros e de sites que o aluno pode visitar; de uma realidade de conceitos, representações e imagens com as quais o aluno passa a lidar e ou vão ajudar a desenvolver outras habilidades, capacidades, comportamentos e até processos cognitivos que a escola tradicional não previa e que o mundo pós-moderno já exige. Além disso, os conteúdos que chegam pela internet tornam-se mais interessantes e atraentes do que quando apresentados em livros ou apostilas, material já tão conhecido pelos alunos; aprender pode tornar-se algo divertido, realístico e mais significativo. A utilização das Tecnologias de Informação e Comunicação promove o empenho dos alunos pelos conteúdos, já que discorrendo sobre diversas tecnologias digitais, consequentemente de novas linguagens, que estão incluídas no dia a dia dos alunos e das escolas. Os estudantes encontram-se com o 
pensamento estruturado pela maneira de representação propiciada pelas novas tecnologias. Desta forma, aderir e empregar estas novas tecnologias possibilita uma aproximação com as gerações presentes nas escolas (ALMEIDA, 2012).

\section{O que é adequado aos adolescentes acessarem na internet?}

Atualmente, entende-se a internet como a principal ferramenta utilizada pelos jovens que estão procurando conhecimento e lazer. A internet na parte do conhecimento é muito útil, pois podemos encontrar conteúdos do mundo inteiro em apenas um "click", ajudando os internautas desde um trabalho escolar até uma tese de doutorado, muitas vezes, rico em detalhes e sempre atualizado.

Na parte do lazer, encontra-se uma internet rica em músicas, informações, artes, etc. Mas o que é mais utilizado na internet por esse público, são as redes sociais, onde é possível encontrarmos pessoas que não vemos há tempo, compartilhar idéias, trocar informações e até mesmo procurar emprego.

\section{A internet dita o comportamento dos adolescentes.}

A adolescência é uma fase do desenvolvimento humano marcada pela transição da infância para a vida adulta, caracterizada por mudanças nos aspectos físico, emocional e social. Aberastury e Knobel (1981) dizem que as modificações corporais, bem como as exigências do mundo externo, demandam novas formas de convivência da parte do jovem que são vivenciadas, a princípio, como uma invasão. Assim, esta etapa do ciclo vital passa a ser um período de construção de uma nova identidade, sendo uma fase de maior susceptibilidade às influências externas.

No dias atuais, os meios de comunicação vêm sendo cada vez mais utilizados, em especial a Internet. Em função disso, há uma preocupação de como ela está sendo usada pelos adolescentes, que representam boa parte dos usuários. Faz-se necessário analisar o comportamento destes jovens e o quanto a Internet influencia a conduta destes.

Esta fase do desenvolvimento é mais apta a sofrer os impactos de uma "realidade frustrante", levando à reflexão de que o tempo que o adolescente dedica à rede poderia ser uma forma de fuga ou esquiva da realidade que não está sendo boa para ele, ou mesmo que esteja, acaba encontrando neste mundo virtual mais reforçadores do que em sua realidade (1981).

Id en line Revista de Psicologia. Ano 7, No. 20, Julho/2013 - ISSN 1981-1179.

Edição eletrônica em http://idonline.emnuvens.com.br/id 
Segundo Barossi, Meira, Góes e Abreu (2009) é frequente a narrativa de pais ou responsáveis por adolescentes sobre a influência do uso excessivo da Internet em seus filhos, assim como a mudança no comportamento demonstrado no dia-a-dia, refletindo em todos os aspectos da vida destes. Incluem-se alterações no humor, comportamento depressivo e reações emocionais explosivas quando são limitados em relação ao uso da rede mundial de computadores.

Vantagens e desvantagens constatadas na utilização da tecnologia na educação dos adolescentes.

Atualmente, ouve-se com freqüência questionamentos sobre os jovens e a tecnologia a respeito do que realmente é verdade quando se trata deste assunto. È freqüente a preocupação dos pais quanto ao tempo que o filho disponibiliza em frente ao computador ou em jogos eletrônicos e celulares. É comum perceber a facilidade com que as crianças lidam com estas tecnologias e, quando se tem alguma dúvida sobre como utilizar alguma ferramenta do celular, é só solicitar ajuda para uma criança e pode-se perceber a facilidade com que ela irá ajudar e mostrar opções que nem mesmo se tem conhecimento. Mas até que ponto isto é positivo? Acredita-se que em tudo existe um lado positivo e negativo, não se pode deixar as crianças disporem demais do seu tempo, vivendo em um mundo tecnológico e deixando de desenvolver outras habilidades que serão importantes para o seu futuro, inclusive o profissional. Tais resultados são apresentado em uma pesquisa realizada por Rosini, Alessandro Marco (s.d.), em que mostraram as vantagens do uso das tecnologias: aumento da criatividade; despertar da curiosidade; especialmente nos casos de utilização no auxílio à aprendizagem de crianças com necessidades especiais. Além disso, trata-se de uma ferramenta rica, auxiliar da aprendizagem, pois disponibiliza a utilização de softwares educacionais (multimídia); Os autores citam como desvantagens: o despreparo dos próprios educadores e alunos, assim como as influências negativas ocasionadas pela utilização de técnicas relacionadas com a tecnologia (computadores). Por fim, Exemplifica, que a utilização excessiva dos instrumentos e se realmente a utilização da tecnologia (computadores) expressará um aperfeiçoamento efetivo do ensino.

$\mathrm{O}$ adolescente desenvolve as habilidades lingüísticas, geográficas e interpessoais. Estas habilidades são desenvolvidas através das diversas possibilidades de aprendizagem com as ferramentas que ficam disponibilizadas na internet $\mathrm{e}$ nos equipamentos que possuem tecnologias atualizadas. As ferramentas de comunicação como MSN, ORKUT, entre outras, ficam responsável pelo desenvolvimento da habilidade interpessoal, onde se torna possível a 
comunicação com pessoas e com novas culturas. Dentro da sala de aula o aluno desenvolve a aprendizagem cooperativa, a pesquisa em grupo, a troca de resultados, conhecimentos e experiências criando círculos de amizades. Uma comunicação bem sucedida aumenta o nível de aprendizado, crescimento e desenvolvimento do aluno.

Segundo Piaget (1975), Sabemos, é verdade, que pelo simples fato de uma criança olhar e manipular um computador, pode levá-la a ter um certo impacto num primeiro momento, levando em alguns casos a alterações no quadro psicológico, pois o tratamento é feito com a máquina através de um processo mecanicista e artificial e não através do relacionamento com outros seres humanos.

Algumas das principais vantagens sobre a utilização de tecnologia na área da educação são: aumento e desenvolvimento da curiosidade, aumento da criatividade que são fatores de suma importância no aprendizado. As principais desvantagens são: educadores despreparados par lidar com as tecnologias, onde em alguns casos ocorre a perda da qualidade, pois alguns educadores acreditam que o aluno estando em frente ao computador, ele esteja desenvolvendo a as habilidades que estão disponíveis para ser aguçadas. Quando realmente o é que preciso que os alunos tenham a instrução de um guia para auxiliar o aluno mostrando as melhores formas de acessar redes sociais para comunicação entre alunos, para realização de atividade curricular escolar, mostrar ferramentas que possam ser utilizadas para realização de trabalhos escolares, assim tendo maior qualidade no aprendizado (PHILLIPS, 2007).

Buelga e Pons (2012) relatam o que vem ocorrendo mundialmente, como uma desvantagem da utilização das tecnologias de informação, o Cyberbullying entre adolescentes, a prevalência e características dos agressores. Juntamente com as formas tradicionais de intimidação, entre iguais, vem surgindo e aumentando no mundo todo, ultimamente, uma nova forma de intimidação e maus tratos entre adolescentes. Estas ocorrências vêm sendo realizadas através das nvas tecnologias de informação e de comunicação, que pressupõe a utilização por parte de um grupo ou sujeito, por meios eletrônicos. Tais como telefones móveis, blogs e páginas da web, e-mails, redes sociais para intimidar de forma deliberada, com ataques pessoais, difamações e /ou outros tipos de intimidação. Com o aumento da disponibilidade de telefones móveis, acesso a Internet, proporciona novos caminhos que alguns adolescentes utilizam para causar danos e angústia a seus pares (García-Pérez, 2011; Keith y Martin, 2005). Especificamente como cyberbullying, tem sido denominada na literatura científica, esta nova forma de assédio realizada através da tecnologia (CAMPBELL, 2005; GENTA, BRIGHI \& GUARINI, 2009; KATZER, FETCHENHAUER \& BELSCHAK, 2009; PATCHIN \& HINDUJA, 2006; SMITH et al.., 2008; WILLARD, 2007).

Id en line Revista de Psicologia. Ano 7, No. 20, Julho/2013 - ISSN 1981-1179. 
Computadores e da Internet têm o potencial de ser usada para fornecer tratamentos psicológicos. Este artigo fornece uma revisão seletiva de aplicações envolvendo pouco ou nenhum contato terapeuta, as aplicações que envolvem a comunicação assíncrona e síncrona com os fornecedores, e as aplicações que têm sido utilizados como coadjuvantes à psicoterapia padrão. Os dados sobre a eficácia destas aplicações mais fortemente apoiar o uso da Internet para preencher e enviar atribuições comportamentais, para obter apoio social de seus pares, e de receber educação, feedback, e apoio de terapeutas na forma de e-mail ou comunicações chatroom. Recomendações práticas gerais são dadas com ênfase em formas de incentivar a utilização da Internet como ferramenta de informação e comunicação como um adjuvante à psicoterapia regular (PHILLIPS, 2007).

A internet oferece a possibilidade de acesso a ambientes sociais voltados para uma comunicação mais rápida e instantânea, ao mesmo tempo as redes sociais podem oferecer uma oportunidade mundial aos seus usuários. As redes globais permitem que os adolescentes se conectem a varias pessoas de várias etnias. Tal comportamento faz com impulsione o desenvolvimento da juventude no que se refere a comunicação, liderança, socialização, informação e aprender em escala internacional. Os adolescentes usam a Internet como fonte de informações de saúde com o intuito de obter as informações necessárias no âmbito sexual e problemas de saúde, que eles provavelmente não têm acesso no local onde vivem (CASSELL et al, 2006).

Nos últimos anos, em uma pesquisa recente mostraram que os estudos das ramificações da internet aumentaram muito. Em dois simpósios sobre pesquisas e teorias que examinam os efeitos de padrões de comportamento em comparação com apenas uma apresentação de pôster no ano anterior. Uma nova revista sobre a internet e dependência está sendo criada, e que a partir do esforço coletivo através de pesquisas poderá se ter uma forma mais adequada de intervenção. O vício em internet pode ser reconhecido como um transtorno legítimo de controle de impulsos digno de sua própria classificação em futuras revisões do Manual Diagnóstico e Estatístico de Transtornos Mentais - DSM (IV-TR). Com a crescente popularidade da Internet, profissionais de saúde mental deverão preparar-se para um aumento da procura de tratamento especificamente concebido para cuidar do paciente com dependência da Internet. Como este vício é novo, as pessoas relutam para buscar tratamento com receio de que os psicólogos não levem suas queixas a sério. Para drogas e álcool existem centros de saúde mental e comunitária, centros de reabilitação e profissionais privados para minimizar o sofrimento e o impacto para os pacientes envolvidos com a internet. Salienta-se a importância de buscar programas de recuperação mais eficazes para esta sintomatologia. Diante disso, para prosseguir esses 
programas de recuperação de efetivos, a continuação da investigação é essencial para melhorar compreender as motivações subjacentes à dependência de Internet (PHILLIPS, 2007).

Pesquisas futuras devem se concentrar em como doença psiquiátrica, como depressão ou transtorno obsessivo compulsivo pode desempenhar um papel no desenvolvimento da utilização da Internet patológico. Estudos longitudinais de viciados em Internet pode revelar traços de personalidade como família, dinâmica, ou habilidades de comunicação influenciam a forma como as pessoas utilizam a Internet. Por último, estudos de resultados são necessários para determinar a eficácia de vários

\section{Conclusões}

O presente artigo estudou sobre os mitos e verdades referente à relação da tecnologia e os adolescentes estudantes do ensino fundamental e médio. Sendo assim, objetivou avaliar em quais aspectos essa relação tornou-se positiva ou negativa no comportamento educacional, social e familiar dos mesmos.

Os resultados mostraram qualitativamente a relevância positiva que a internet, dentre outras tecnologias, tem apresentado, abrindo um mundo de possibilidades aos estudantes da era digital. Falamos de uma infinidade de livros e de sites que o aluno pode visitar; de uma realidade de conceitos, representações e imagens com as quais o aluno passa a lidar que irá ajudá-los no desenvolvimento de outras habilidades, capacidades, comportamentos e até processos cognitivos que a escola tradicional não previa e que o mundo pós-moderno já exige dele. Além disso, os conteúdos que chegam pela internet tornam-se mais interessantes e atraentes do que quando apresentados em livros ou apostilas, material já tão conhecido pelos alunos; aprender pode tornar-se algo divertido, realístico e mais significativo.

Em pesquisas recentes observa-se que com o aumento da disponibilidade de telefones móveis, acesso a Internet, proporciona novos caminhos que alguns adolescentes utilizam para causar danos e angústia a seus pares. Especificamente como cyberbullying, tem sido denominada na literatura científica, esta nova forma de assédio realizada através da tecnologia, vem trazendo prejuízos para a população, principalmente para os pares de adolescentes.

Diante desse contexto, ressalta-se que a tecnologia aliada à internet e às escolas, exercerá um papel de suma importância para o desenvolvimento de habilidades que irão influenciar positivamente na vida escolar não apenas dos adolescentes, mas das crianças, jovens e adultos. Com essa integração, tendo em vista a abrangência instantânea que a mesma proporciona diante daqueles que utilizam este método de forma consciente, será possível que os

Id en line Revista de Psicologia. Ano 7, No. 20, Julho/2013 - ISSN 1981-1179. Edição eletrônica em http://idonline.emnuvens.com.br/id 
alunos agreguem novos conhecimentos que trarão resultados positivos para o seu rendimento escolar.

Faz-se necessário, diante deste estudo, uma pesquisa quantitativa para que se possa entender melhor este fenômeno. Sugere-se um estudo com método quantitativo com adolescentes estudantes das escolas de ensino fundamental e médio, para que seja apresentada em números a relevância da implantação da tecnologia nas salas de aula, bem como o seu bom aproveitamento na jornada escolar dos alunos.

\section{Referências}

ABERASTURY, A.; KNOBEL, M. Adolescência normal: um enfoque psicanalítico. Tradução de Suzana Maria Garagoray Ballve. Porto Alegre: Artmed, 1981.

ALMEIDA, M. E. Tecnologia na Sala de aula. Revista Escola. [s.d]. Disponível em: (http://revistaescola.abril.com.br/). 2012. Acesso em 15 jan.

BAROSSI, O.; MEIRA, S. V. E.; GÓES, D. S.; ABREU, C. N. Carta aos editores Programa de Orientação a Pais de Adolescentes Dependentes de Internet (PROPADI). Revista Brasileira de Psiquiatria. São Paulo - SP. Carta_689_V01.indd 131.08 .09 10:48:30. 2009. Disponível em: http://submission.scielo.br/index. php/rbp/article/view/13161/1559. Acesso em: 17 nov. de 2010.

BUELGA, S. \& PONS, J. Agresiones entre Adolescentes a través del Teléfono Móvily de Internet. Psychosocial Intervention. Vol. 21, No. 1, 2012. pp. 91-101.

CAMPBELL, M. A. Cyberbullying: An old problem in a new guise? Australian Journal of Guidance and Counselling, 15, 2005. 68-76.

CASSELL, J., HUFFAKER, D., TVERSKY, D., \& FERRIMAN, K. (2006). The language of online leadership: Gender and youth engagement on the Internet. Applied Developmental Psychology, 42, 436-449

COSTA, Sérgio Roberto (Hiper) textos ciberespaciais: mutações do/no lerescrever. Caderno Cedes, vol. 25, no 65, 2005. pp.102-116.

Id en line Revista de Psicologia. Ano 7, No. 20, Julho/2013 - ISSN 1981-1179. Edição eletrônica em http://idonline.emnuvens.com.br/id 
FRANK, Scott, diretor da universidade Case Western Reserve. Adolescente que abusa da tecnologia prejudica saúde. Nov.2011. [s.l.] Disponível em: http://www.cabecadecuia.com/noticias/84365/adolescente-que-abusa-da-tecnologia-prejudicasaude-diz-estudo.html

GENTA, M. L., BRIGHI, A. \& GUARINI, A. European project on bullying and cyberbullying granted by Daphne II Programme. Journal of Psychology, 217, 2009. 233-234.

GIL, A.C. Métodos e Técnicas de Pesquisa Social. São Paulo. $5^{a}$ edição. Ed. Altas S.A, 2007.

KATZER, C., FETCHENHAUER, D. \& BELSCHAK, F. Cyberbullying: Who are the victims? A comparison of victimization in internet chatrooms and victimization in school. Journal of Media Psychology, 21, 2009. 25-36.

KENSKI, V. M. Educação e Tecnologias - O Novo Ritmo Da Informação. São Paulo: Papirus, 2003.

Tecnologias e Ensino Presencial e a Distância. Campinas, SP: Papirus, 2008.

MARCUSCHI, Apud GOMES, Ana Lúcia et al 2005 Escrita teclada x escrita padrão na produção textual: a experiência de adolescentes brasileiros. Disponível http://www.scielo.oces.mctes.pt/pdf/rpe/v22n1/v22n1a04.pdf . Escrita teclada x escrita padrão na produção textual: a experiência de adolescentes brasileiros Revista Portuguesa de Educação, 2009, 22(1), pp. 71-88.

PATCHIN, J. \& HINDUJA, S. Bullies move beyond the schoolyard: A preliminary look at cyberbullying. Youth Violence and Juvenile Justice, 4, 2006. 148-169.

PHILlIPS, M. Internet Dependency (Addiction) - Some Initial Research. Ann Arbor Publishers. Disponível no endereço eletrônico; http://www.annarbor.co.uk/images/PDF/InternetAddiction.pdf. Acesso em 06-02-2012. 2007. 
PHILLIPS, M. Internet dependency (addiction) - some initial research. Ann Arbor Publishers, 2007.

ROSINI, A. M. O uso da tecnologia da informática na educação, Uma reflexão no ensino com crianças[s.d].Mestre em Administração de Empresas e Doutorando em Comunicação e Semiótica pela PUCSP. Consultor em Tecnologia e Sistemas de Informação.

SMITH, P. K, MAHDAVI, J., CARVALHO, C., FISHER, S., RUSSELL, S. \& TIPPETT, N. Cyberbullying: Its Nature And Impactin Secondary School Pupils. Journal Of Child Psychology And Psychiatry, 49, 2008. 376-385.

SMITH, P. K, MAHDAVI, J., CARVALHO, C., FISHER, S., RUSSELL, S. Y TIPPETT, N. (2008). Cyberbullying: Its nature and impact in secondary school pupils. Journal of Child Psychology and Psychiatry, 49, 2008. 376-385.

Como citar este artigo (Formato ISO):

DOMINSKI, D.K.; BRITO, D.E.N.; SANTOS, I.N.; RODRIGUES, J.A.; MOURA, E.; LOPES, R.M.F.; ESTEVES, C.S. Reflexões sobre a tecnologia e adolescentes: mitos e verdades. Id on Line Revista de Psicologia, Julho de 2013, vol.1, n.20, p. 22-32. ISSN 1981-1189. 